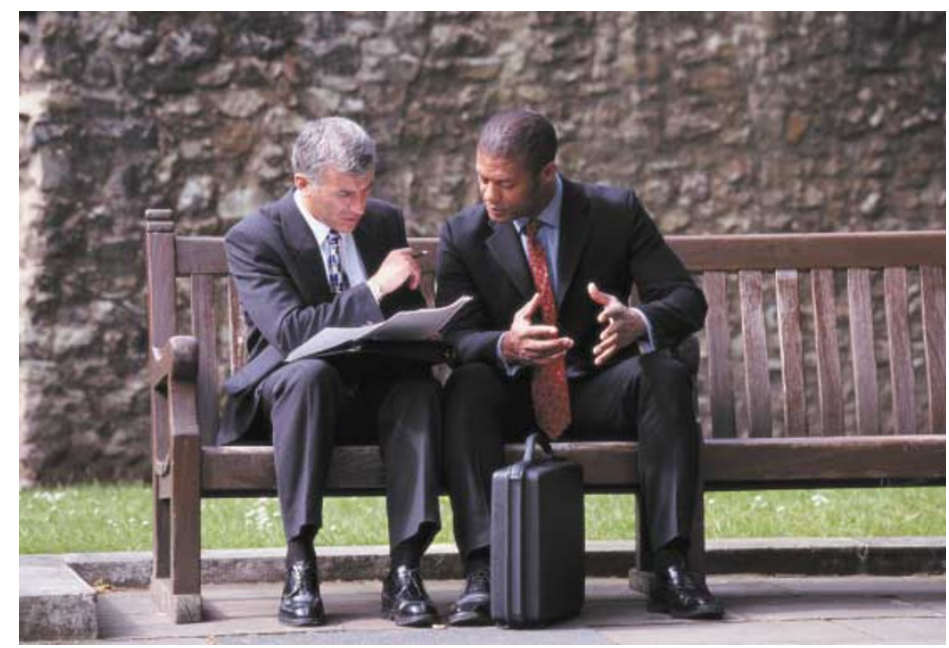

HUMAN EVOLUTION O

\section{Two mutations worth a thousand words (or more)}

It's one of the basic questions of our existence: what makes humans different from our close cousins, the apes? Are we distinct owing to many genetic mutations or, as some have postulated, just a few key base changes? A new study by Enard et al. in Nature suggests that two aminoacid mutations in one gene might have contributed greatly to the evolution of modern humans.

One defining trait for humanity is our ability to communicate through language, and thus develop culture over time. In a 2001 Nature paper, Lai et al. reported that mutations in the FOXP2 gene impaired some humans' ability to speak and to use language properly. The FOXP2 protein is a forkhead transcription factor that presumably controls the expression of other genes. So, it might seem like a perfect lead to the biological basis of that unique trait - the use of language.

This discovery led Enard et al. to scrutinize FOXP2 sequence for polymorphisms in humans and between humans and other species. Although no amino-acid polymorphisms were found in various human populations, all humans have two aminoacid changes that are specific to our lineage, and that are not shared with either four ape species or our distant mouse relatives. Furthermore, one of these changes might be functionally important, as it creates a target site for phosphorylation on, and therefore regulation of, the FOXP2 transcription factor protein.

When Enard et al. studied nucleotide polymorphisms in
FOXP2 they found that the pattern of polymorphisms suggests that it is the genomic region that has experienced strong positive selection. Amazingly, selection has been mainreported that this chromosomal region undergoes recombination at five times the genomic average. As the nearest gene is $286 \mathrm{~kb}$ away, the most likely explanation is that FOXP2 itself is the target of the positive selection.

Finally, Enard et al. used evolutionary modelling to speculate that the human-specific changes occurred at the time of emergence of anatomically modern humans. So, they propose that the development of language, a key factor in allowing early humans to migrate and spread to new territories, was aided by key changes in FOXP2, which quickly swept through the human population. Although other genes are certainly involved in the process, Enard et al. have set forth a blueprint for how to determine the roots of our human family tree, one gene at a time.

Chris Gunter, Associate Editor, Nature

\section{(20) References and links} ORIGINAL RESEARCH PAPERS Enard, W. et al. Molecular evolution of $F O X P 2$, a gene involved in speech and language. Nature 418, 869-872 (2002) | Newbury, D. F. et al. FOXP2 is not a major susceptibility gene for autism or specific language impairment. Am. J. Hum. Genet. 70, 1318-1327 (2002)

FURTHER READING Lai, C. S. et al. A forkheaddomain gene is mutated in a severe speech and language disorder. Nature 413, 519-523 (2001) WEB SITE

Svante Paabo's lab:

http://www.eva.mpg.de/genetics tained even though Newbury et al.

\section{IN BRIEF}

\section{TECHNOLOGY}

New genes involved in cancer identified by retroviral tagging.

Suzuki, T. et al. Nature Genet. 19 Aug 2002 (doi:10.1038/ng949)

High-throughput retroviral tagging to identify components of specific signalling pathways in cancer.

Mikkers, H. et al. Nature Genet. 19 Aug 2002 (doi:10.1038/ng950)

Genome-wide retroviral insertional tagging of genes involved in cancer in Cdkn2a-deficient mice.

Lund, A. H. et al. Nature Genet. 19 Aug 2002 (doi:10.1038/ng956)

Three groups report here that a modification of the invertebrate suppressor/enhancer screen can be used successfully in mammals. To demonstrate this, they infected mice already mutant at certain loci - loci that are known to cause lymphomas - with Moloney murine leukemia virus, which accelerates and exacerbates lymphoma formation. The resulting tumours were screened for common insertion sites (CISs) that were then cloned and compared against the assembled mouse genome sequence to identify the disrupted loci. The screen yielded many known and novel loci that synergize with the initial mutation to bring about tumorigenesis. Because each group started with a different mouse mutant, most of the obtained CISs were unique, but common loci (mostly genes involved in cell proliferation and differentiation) were also recovered, validating the specificity of this approach.

\section{MOUSE MODELS}

Disruption of Dag1 in differentiated skeletal muscle reveals a role for dystroglycan in muscle regeneration.

Cohn, R. D. et al. Cell 110, 639-648 (2002)

To better understand the pathogenesis of muscular dystrophy (MD) and the functions of dystroglycan (Dag) in mature differentiated skeletal muscle, Cohn et al. used the Cre-loxP system to inactivate Dag specifically in mouse skeletal muscle using the muscle creatine kinase (MCK) promoter. The resulting mutant mice had a surprisingly mild MD phenotype, compared to Dagnull mice and humans, owing to the expression of Dag in the satellite cells of skeletal muscle. Together, the findings of this study show that the inadequate repair of skeletal muscle by satellite cells is an important mechanism in the pathogenesis of MD.

\section{HUMAN GENETICS}

Allelic variation in human gene expression.

Yan, H. et al. Science 297, 1143 (2002)

Here, Yan et al. report a fluorescent dideoxy terminator-based technique to quantify allelic variation in gene expression in normal individuals that are heterozygous for a SNP. Significant differences in allelic expression of 6 of the 13 genes examined were observed. Moreover, altered allelic expression of two of the studied genes was consistently inherited in the families of probands. These findings indicate that cis-acting inherited variations in gene expression are relatively common among normal individuals. 